\section{A) Check for updates}

Cite this: Analyst, 2020, 145, 6600

\title{
Single-molecule brightness analysis for the determination of anticancer drug interactions with DNA $\dagger$
}

\author{
Ying Zhou, (D) \$ Krzysztof Bielec, (D) † Pakorn Pasitsuparoad (D) and Robert Hołyst (D) * \\ Anthracyclines are one of the most studied anticancer drugs approved for medical treatment. The equili- \\ brium constant $(K)$ of the reaction between these drugs with DNA in both in vitro and in vivo experiments \\ lacks consensus. The $K$ values vary from $10^{4}$ up to $10^{8} \mathrm{M}^{-1}$, which suggest a 1000 -fold error in determin- \\ ing the effective concentration needed to form the drug-DNA complex. Until 2014, only one study by \\ García [J. Phys. Chem. B, 2014, 118, 1288-1295] showed that the binding of anthracycline representative \\ doxorubicin occurs in two reactions. We support this result by brightness analysis at a single molecular \\ level for the four most common anthracyclines: doxorubicin, daunorubicin, epirubicin, and idarubicin.
}

Received 3rd June 2020, Accepted 18th July 2020 DOI: 10.1039/d0an01108h rsc.li/analyst step reaction. ${ }^{8}$ However, additional binding of the DOX to the DOX-DNA complex has been recently reviled. ${ }^{2-4,9}$ As both the reactions occur at the same time, the second reaction is mostly observed when the amount of DOX is in large excess to the DNA binding sites. The excess of the DOX molecules attach to the formed DOX-DNA complexes with a weaker binding strength than DOX molecules attached in the first reaction.

Due to the cardio-toxicity and dose resistance of DOX, people prefer to use the less harmful DOX derivatives-daunorubicin (DNR), epirubicin (EPR), and idarubicin (IDR) in cancer treatment. ${ }^{11}$ However, compared with DOX, there are fewer kinetic studies about these anthracycline-DNA interactions. In addition, the reported $K$ values suggest a similar methodological inaccuracy as that in the case of DOX (see Table 2). Considering the structures of these drugs, they are all tetracenequinone-based chromophore groups and possess the positive nitrogen on the sugar moiety. It was inferred that the small structural differences of the hydroxyl groups (Fig. 1) should not significantly affect the $K$ values of these drugs in their reaction with DNA. However, there is still a lack of systematic kinetic studies to compare the kinetics of these four drugs. Will they share the same reaction mechanism with the corresponding $K$ values?

Table 1 Equilibrium constant $(K)$ determined in the DOX-DNA interaction at different ionic strengths $(l)$

\begin{tabular}{lll}
\hline Method & $I(\mathrm{mM})$ & $K\left(\times 10^{6} \mathrm{M}^{-1}\right)$ \\
\hline Fluorescence spectroscopy $^{2}$ & 100 & 0.01 \\
Isothermal calorimetry titration $^{10}$ & 100 & 0.3 \\
Fluorescence correlation spectroscopy $^{6}$ & 10 & 1.0 \\
Fluorescence spectroscopy $^{2}$ & 63 & 13 \\
Fluorescence spectroscopy $^{3}$ & 2.5 & 230
\end{tabular}

Institute of Physical Chemistry, Polish Academy of Sciences, Kasprzaka 44/52, 01-224 Warsaw, Poland. E-mail: rholyst@ichf.edu.pl

$\dagger$ Electronic supplementary information (ESI) available. See DOI: 10.1039/ D0AN01108H

$\$$ These authors contributed equally to this work. 
Table 2 Equilibrium constant $(K)$ determined in the anthracycline-DNA interaction in references

\begin{tabular}{lll}
\hline Drug & Method & $K\left(\times 10^{6} \mathrm{M}^{-1}\right)$ \\
\hline DNR & Resonance light scattering $^{12}$ & 0.02 \\
& Second harmonic generation $^{13}$ & 0.2 \\
& Cyclic voltammetry $^{14}$ & 1.2 \\
EPR & UV-vis spectroscopy $^{15}$ & 0.03 \\
& UV-vis spectroscopy $^{16}$ & 0.4 \\
IDR & Fluorescence spectroscopy $^{17}$ & 0.6 \\
& UV-visible spectroscopy $^{18}$ & 0.02
\end{tabular}

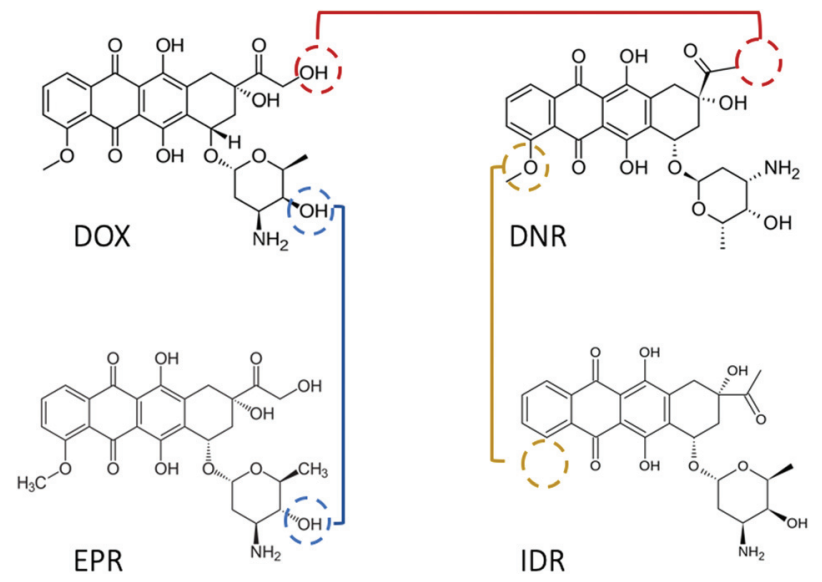

Fig. 1 Structures of the four most important and effective anthracyclines. Doxorubicin and daunorubicin contain a tetracyclic aglycone structure of four cyclohexane chains with a daunosamine sugar moiety. However, daunorubicin is seen with the absence of a hydroxylgroup (red line). Compared with doxorubicin, epirubicin has an opposite chirality of the hydroxyl group in the daunosamine moiety (blue line). Idarubicin is identical to daunorubicin except the lack of 4-methoxy group on the ring (yellow line).

In our previous work, we developed a sensitive and accurate method to determine the equilibrium constant based on the molecular brightness (MB) of a single fluorophore. We have used this approach previously to determine the strength of the interaction between a pair of complementary oligonucleotides labeled with only one dye, even at $100 \mathrm{pM}$ concentration of DNA strands. ${ }^{19}$ We define $\mathrm{MB}$ as the number of photons emitted by a number of molecules (e.g. anticancer drug) inside the confocal volume. After the formation of a drug-DNA complex, its MB could be enhanced or quenched, and thus the number of emitted photons changes. ${ }^{20} K$ was determined using the ratio of the MB of drug molecules at free and bound states at a given concentration of the second substrate. With this approach, even a slight change in the number of emitted photons can be distinguished.

Here, we report a revised mechanism of DOX-, DNR-, EPR-, and IDR-DNA interactions consisting of two reactions, following with the corresponding $K$ in each one. We observed the $\mathrm{MB}$ change of each drug at a single molecular level in the formation of the drug-DNA complexes. We proposed the structural model of interaction in the second reaction by analyzing drug fluorescence anisotropy properties. The determined average $K$ in experiments with DOX binding to the different structures (linear or circular) and lengths of DNA (from $20 \mathrm{bp}$ to $48 \mathrm{kbp})$ are equal to $(6.9 \pm 1.4) \times 10^{7} \mathrm{M}^{-1}$ in the first reaction and $(1.5 \pm 0.6) \times 10^{6} \mathrm{M}^{-1}$ in the second one. By monitoring the MB change of each drug, we proposed the structural binding insights and determined the transition point between each reaction (around 0.4 drug molecules per bp). The determined $K$ values in the first $\left(K_{1}\right)$ and the second reaction $\left(K_{2}\right)$ show around a 40-time difference among all four anthracyclines.

\section{Experimental section}

\subsection{Materials}

Buffer (Tween 20-EDTA-sodium phosphate, $\mathbf{p H}=7.4$ ). The $100 \mathrm{mM}$ stock solution was prepared by diluting $19 \mathrm{~mL}$ of 0.2 $\mathrm{M} \mathrm{NaH} \mathrm{PO}_{4} \cdot \mathrm{H}_{2} \mathrm{O}$ and $81 \mathrm{~mL}$ of $0.2 \mathrm{M} \mathrm{Na}_{2} \mathrm{HPO}_{4} \cdot 7 \mathrm{H}_{2} \mathrm{O}$ with $100 \mathrm{~mL}$ of Milli-Q water. The working buffer was prepared by diluting $146.12 \mathrm{mg}$ of EDTA and $50 \mathrm{~mL}$ of $100 \mathrm{mM}$ sodium phosphate into $450 \mathrm{~mL}$ of distilled water; $0.002 \%$ Tween 20 was used as a surfactant.

Anthracyclines. Solid doxorubicin hydrochloride (DOX) was purchased from Santa Cruz Biotechnology Inc. The DOX stock solution was prepared in Milli-Q water to obtain $100 \mu \mathrm{M}$, then it was sonicated and further aliquoted to $10 \mu \mathrm{M}$. The aliquots were stored at $4{ }^{\circ} \mathrm{C}$. Solid daunorubicin hydrochloride (DNR), epirubicin hydrochloride (EPR), and idarubicin hydrochloride (IDR) were purchased from Sigma-Aldrich, Inc. DNR, EPR, and IDR were prepared in the same way as DOX. Before the experiment, the aliquot was diluted to $80 \mathrm{nM}$ as the working concentration.

DNA. We used different lengths and types of DNA. Oligonucleotide double-strands of length 20 bp and 69 bp were synthesized by IBA GmbH, Germany; NoLimits 2500 bp DNA Fragment, pUC 19 plasmid DNA (2686 bp), and $\lambda$ DNA (48 502 bp) were purchased from Thermo Fisher Scientific, USA; and purified calf thymus DNA (ct DNA, $13200 \mathrm{bp}$ ) was purchased from Merck KGaA, Darmstadt, Germany. All types of DNA were stored in standard Tris-EDTA buffer at $-20{ }^{\circ} \mathrm{C}$. In a typical experiment for $K$ determination, the selected DNA was diluted into a series of working concentrations from $800 \mathrm{nM}$ to $80 \mathrm{nM}$ of bp (in terms of $\mathrm{bp})$; there were 16 concentrations in total. No chemicals were further purified unless specifically stated.

The anthracycline working solution was mixed in equal volumes with the DNA. The mixture was incubated over 12 hours at $25{ }^{\circ} \mathrm{C}$ before measurement. The final concentration of anthracycline in the experiment was set at $40 \mathrm{nM}$ unless explicitly stated otherwise.

\subsection{Microscope setup}

Experiments were carried out on an inverted confocal microscope (Nikon C1). The system was upgraded with a PicoQuant LSM module, including the PicoHarp 300 Time-Correlated Single-Photon Counting setup (TCSPC). The sub-femtolitre detection volume was obtained using a $60 \times(\mathrm{NA}=1.2)$ water 
immersion objective (Nikon Plan Apo). A pulsed blue (485 nm) diode laser (PicoQuant $\mathrm{GmbH}$, Germany) with a frequency of $40 \mathrm{MHz}$ was used as the light source. To prevent photobleaching of DOX, we measured the laser power by the power meter (PM 100, Thorlabs) and set it at $50 \pm 5 \mu \mathrm{W}$. The samples were loaded in a glass-bottom container (Thermo Scientific ${ }^{\mathrm{TM}}$ Lab-Tek $^{\mathrm{TM}}$ chamber slide), the temperature was controlled at $25 \pm 0.5{ }^{\circ} \mathrm{C}$ within a climate chamber (OkoLab, Italy). The focal volume was set at a distance of $10 \mu \mathrm{m}$ from the edge of the cover glass. The fluorescence signal was filtered by the 488 long-pass filter and collected by two Single Photon Avalanche Diodes (MPD and PerkinElmer). Each experiment was preceded by a calibration using rhodamine 110 (Sigma-Aldrich). Typically, a single measurement lasted 90 seconds. The control of the system and prepossessed data analysis was dealt with by the Symphotime 64 software. Further data analysis was performed by self-written Python scripts.

\subsection{Brightness method}

We explored drug-DNA complex formation by analyzing the $\mathrm{MB}$ of a single fluorophore. Anthracyclines are known to possess poor fluorescence properties. We define $\mathrm{MB}$ as the number of photons emitted per second per fluorophore. To describe the brightness method, we use the equations in which the substrates are administered in molar concentration. Hence, in equations, $N_{\mathrm{A}}$ is the Avogadro constant. In the example of a pure drug solution (where "drug" can be DOX, DNR, etc.), in the given volume $\left(V_{0}\right)$, the average number of photons emitted per second (count rate, $I_{0}$ ) is proportional to drug concentration $\left(c_{\text {drug }}\right)$ and its intrinsic $\mathrm{MB}(\alpha)$. There is:

$$
V_{0} \cdot N_{\mathrm{A}} \cdot c_{\text {drug }} \cdot \alpha=N_{\text {drug }} \cdot \alpha=I_{0}
$$

When DNA is added into the drug solution, the drug molecules intercalate into the bp of DNA.

$$
\mathrm{drug}+\mathrm{DNA} \stackrel{K_{1}}{\rightleftharpoons} \mathrm{drug}-\mathrm{DNA}
$$

The fluorescent components in the system are the drug molecules and drug-DNA complexes. Now, the measured number of photons is $I_{1}$. The drug-DNA complexes have an intrinsic $\mathrm{MB} \gamma_{1}$, which is different from that of drug $\operatorname{MB}(\alpha)$. Therefore, eqn (1) changes to:

$$
V_{0} \cdot N_{\mathrm{A}} \cdot\left(\alpha \cdot c_{\mathrm{drug}}^{\mathrm{eq}}+\gamma_{1} \cdot c_{\mathrm{drug}-\mathrm{DNA}}^{\mathrm{eq}}\right)=I_{1}
$$

The equilibrium constant in this reaction $\left(K_{1}\right)$ is given by:

$$
K_{1}=\frac{c_{\mathrm{drug}-\mathrm{DNA}}^{\mathrm{eq}}}{c_{\mathrm{drug}}^{\mathrm{eq}} \cdot c_{\mathrm{DNA}}^{\mathrm{eq}}}
$$

After transformation, $I_{1}$ can be re-written as:

$$
\begin{aligned}
V_{0} \cdot N_{\mathrm{A}} \cdot \alpha \cdot\left[c_{\mathrm{drug}}-c_{\mathrm{drug}-\mathrm{DNA}}^{\mathrm{eq}}\right] \\
\cdot\left[1+\frac{\gamma_{1}}{\alpha} \cdot K_{1} \cdot\left(c_{\mathrm{DNA}}-c_{\mathrm{drug}-\mathrm{DNA}}^{\mathrm{eq}}\right)\right]=I_{1}
\end{aligned}
$$

Recent works show that besides a simple intercalation of DOX to DNA, there is a second interaction mechanism. ${ }^{3,4,9}$
The second reaction mechanism is the formation of the external complexes between the DOX molecules and the formed DOX-DNA complexes. The impact of this reaction becomes apparent when all the available binding sites on the DNA chain are occupied by DOX molecules, although they occur at the same time. ${ }^{3}$ The second reaction can be illustrated as:

$$
\operatorname{drug}+\operatorname{drug}-\mathrm{DNA} \stackrel{K_{2}}{\rightleftharpoons} \operatorname{drug}-(\mathrm{drug}-\mathrm{DNA})
$$

The drug-(drug-DNA) complexes formed in the second reaction have an intrinsic $\mathrm{MB} \gamma_{2}$, which is different from $\alpha$ and $\gamma_{1}$. In this case, after the addition of the DNA, three fluorescent components can be present in the reaction system (there is no formation of transition state), but the count rate $I_{1}$ is still the same. Thus, eqn (2) can be written as:

$$
\begin{gathered}
V_{0} \cdot N_{\mathrm{A}} \cdot\left(\alpha \cdot c_{\mathrm{drug}}^{\mathrm{eq}}+\gamma_{1} \cdot c_{\mathrm{drug}-\mathrm{DNA}}^{\mathrm{eq}}\right. \\
\left.+\gamma_{2} \cdot c_{\mathrm{drug}-(\mathrm{drug}-\mathrm{DNA})}^{\mathrm{eq}}\right)=I_{1}
\end{gathered}
$$

The equilibrium constant in the second reaction $\left(K_{2}\right)$ is given by:

$$
K_{2}=\frac{c_{\mathrm{drug}-(\mathrm{drug}-\mathrm{DNA})}^{\mathrm{eq}}}{c_{\mathrm{drug}}^{\mathrm{eq}} \cdot c_{(\mathrm{drug}-\mathrm{DNA})}^{\mathrm{eq}}}
$$

Finally, $I_{1}$ can be transformed into:

$$
\begin{aligned}
& V_{0} \cdot N_{\mathrm{A}} \cdot \alpha\left[\left(c_{\mathrm{drug}}-c_{\mathrm{drug}-\mathrm{DNA}}^{\mathrm{eq}}\right)\right. \\
& \cdot\left(1+\left(\frac{\gamma_{1}}{\alpha}\right) \cdot K_{1} \cdot\left(c_{\mathrm{DNA}}-c_{\mathrm{drug}-\mathrm{DNA}}^{\mathrm{eq}}\right)\right. \\
&\left.\left.+\left(\frac{\gamma_{2}}{\alpha}\right) \cdot K_{2} \cdot c_{\mathrm{drug}-\mathrm{DNA}}^{\mathrm{eq}}\right)\right]=I_{1}
\end{aligned}
$$

In the ESI, $\dagger$ we have described all of the steps to obtain eqn (3) and (5) in detail. To determine $K_{1}$ and $K_{2}$ in eqn (5), we measured the $\mathrm{MB}$ of each component $\left(\alpha, \gamma_{1}\right.$, and $\left.\gamma_{2}\right)$. More specifically, (1) $\alpha$ is determined in one FCS experiment (see Fig. 2, the details can be seen in our previous work $\left.{ }^{6}\right)$; (2) $\gamma_{1}$ is evaluated in a titration experiment when $c_{\mathrm{DNA}} \gg c_{\mathrm{drug}}$; (3) $\gamma_{2}$ is evaluated by a titration experiment when $c_{\mathrm{DNA}} \ll c_{\mathrm{drug}}$.

\section{Results and discussion}

\subsection{Proof of two equilibrium states in DOX-DNA interaction}

To explore the interaction mechanism between the drug and DNA, we started with the most widely studied anthracyclineDOX interaction. We monitored the DOX MB change in the reaction with a 69 bp double-stranded DNA. In the titration experiment, we kept the DOX concentration constant and varied the DNA concentration (in terms of bp concentration). In Fig. 3, we present MB as a function of DOX to DNA ratio $(R)$. By fitting the $\mathrm{MB}$ change with a linear curve, we determined the cross-over point $(R=0.39 \pm 0.05)$ of the two regimes.

The average bp number per binding site is $3.1 .^{21} \mathrm{We}$ expected to reach the saturation of the available binding sites 


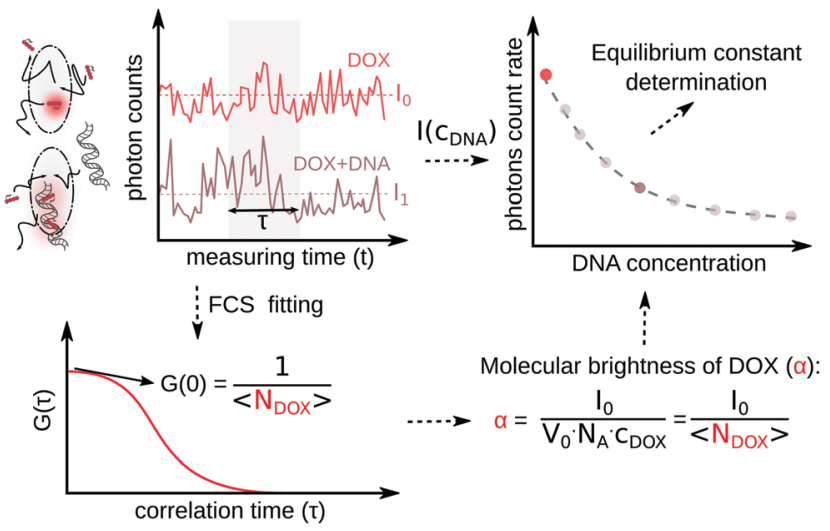

Fig. 2 Scheme of molecular brightness determination. In a confocal volume $\left(V_{0}\right)$, the photon counts in a time $(t)$ are recorded by the detector. In a single measurement, by dividing the photon counts with $(t)$ for a given concentration of DOX, the photon count rate $\left(I_{0}\right)$ of the DOX molecules is known. Next, an autocorrelation curve will be derived from the same photon count curve in FCS measurement, and with proper fitting, the number of DOX molecules $\left(N_{\text {DOX }}\right)$ inside the confocal is calculated. With the obtained parameter $I_{0}$ and $N_{\mathrm{DOX}}, \alpha$ is calculated. With the addition of DNA, $I_{0}$ will decrease to $I_{1}$; by dividing $I_{1}$ with $N_{\text {DOx }}$ at a specific DNA concentration, $\gamma_{1}$ and $\gamma_{2}$ can be calculated.

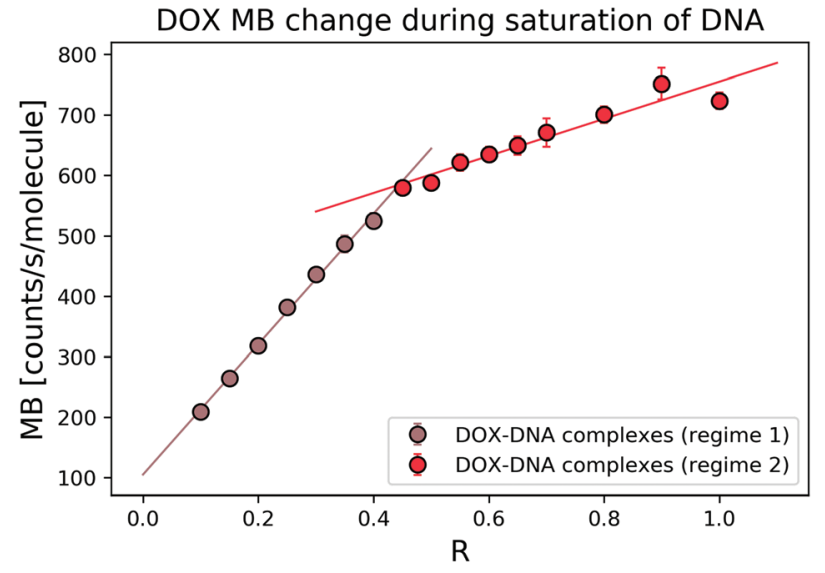

Fig. 3 DOX average MB change in the reaction with 69 bp DNA. With the increase of $R$, it is possible to determine the two regimes where DOX has different trends in MB change. Free DOX molecules have an MB $(\alpha)$ of 1025 counts per s per molecule in this specific measurement.

when $R$ was 0.32 . Thus, we focused on the regime where $0<R$ $\leq 1$ to determine the MB of the formed complexes. To determine the $\mathrm{MB}$ of each complex, we set the intercept of the linear fitting as DOX MB in each regime. In the first regime $(R<0.4)$, DOX lost $8.36 \pm 1.92$ fold of its initial MB, while in the second regime $(R>0.4)$, we observed a $2.28 \pm 0.01$-fold decrease. Our determined cross-over point $(R=0.39 \pm 0.05)$ of the two regimes was in good relation with the previously reported number of $0.35 .^{3}$

The DOX MB loss in the first regime $(R<0.4)$ can be explained as follows: when there are enough binding sites on the DNA chain, the aromatic group of DOX molecules will intercalate into the DNA bp through $\pi$-stacking interactions. ${ }^{22}$ Due to the stacking, the motion of the DOX aromatic groups is fully limited, leading to a dramatic fluorescence quenching. ${ }^{20}$ Meanwhile, two possible mechanisms can explain the smaller DOX MB loss in the second regime $(R>0.4)$. The first hypothesis is chemical interaction. In the formation of a second complex, the DOX molecules bind to the occupied binding sites with a similar conformation as that in the first reaction, ${ }^{4}$ although the influence on MB of DNA is lower as the first molecule compensates most of the initial interactions. In the second hypothesis, we infer that the chromophore group of the DOX molecules did not participate in the interaction as actively as that in the first reaction. Previous reports suggest an interaction between the positive N3' nitrogen on the amino sugar of the DOX molecule and the backbone of the negatively charged DNA. ${ }^{2-4,9}$ Based on the rotation hypothesis, we assume that in the second mechanism, the aminoglycoside ring of the DOX molecules will interact electrostatically with DNA. In such a model (Fig. 4c), the rotational motion of the chromophore group in the DOX molecules is only partially limited; thus, the DOX molecules can emit more photons (the MB loss is less). ${ }^{9,23}$

To find out which hypothesis in the second interaction mechanism is more accurate, we performed the brightness

a)

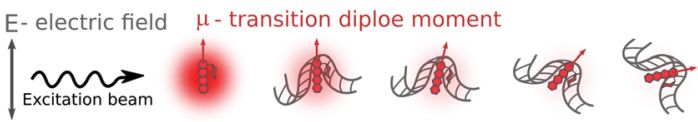

b)

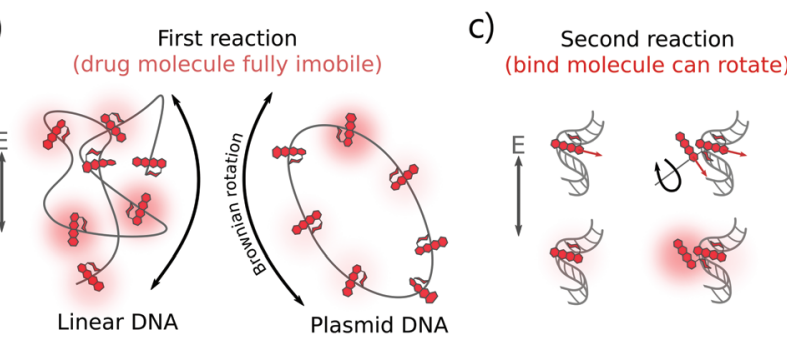

Fig. 4 Explanation of DOX MB change in the binding with DNA. (a) The photon absorption probability of the fluorophores (here refers to DOX molecules) depends on the angle between the transition dipole moment of the fluorophore and the electric field vector of the excitation light. Frequent emission is possible from free DOX molecules because they have a fast rotation. After binding with DNA, the DOX energy levels are affected by the DNA and hence DOX MB then changes. (b) Another factor that affects DOX MB change is the physical origin of the formed complexes. Once the complexes are formed, the direction of the drug dipole moment vector depends on the orientation of the attached DNA. Brownian rotation of DNA dictates the dipole vectors of the bound DOX molecules. Entangled linear DNA possesses more isotropic distribution of dipole vectors than the circular plasmid DNA. Circular DNA is less prone to twisting or bending, resulting in the bound DOX being more dependent on the rotation of plasmid DNA in space. (c) During the formation of the second complex, the bound DOX MB is less dependent on the DNA structure because the chromophore group of the DOX molecules has not participated in the reaction and it has more freedom to rotate in the electric field. As a result, the bound DOX in this complex is much brighter than that in the first reaction. 


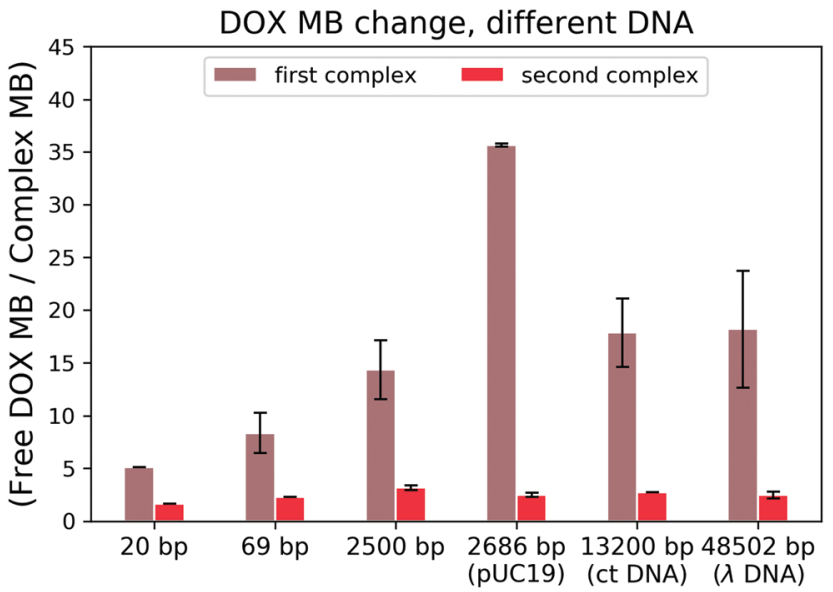

Fig. 5 DOX MB loss in the reaction with DNA.

analysis with different types of DNA: from the very short $20 \mathrm{bp}$ DNA to the very long 48k bp DNA and from the linear $2500 \mathrm{bp}$ DNA to 2686 bp circular plasmid DNA (see Fig. 5). We calculated the relative DOX MB loss as the ratio of free DOX MB to the formed complex MB in each interaction. The larger the value, the more the MB loss. On average, the DOX molecules lost $16.56 \pm 10.70$ fold of the initial MB during the formation of the first complex and $2.45 \pm 0.50$ fold in the second one.

There is a correlation between the length of DNA and $\mathrm{MB}$ (the longer the DNA, the more significant the MB loss) in the first reaction, except for circular DNA. The MB loss of the second complex was similar irrespective of the DNA length or structure. The structure of the DOX molecules does not allow to transform it into other quenched conformations such as the case of Cy3 dye (trans- to cis-isomerization). The phenomenon in the first reaction can be explained as follows: when the DOX-DNA complexes are formed, the DOX molecules are trapped in the DNA backbone. With the increase in the number of the negatively charged bps in the DNA backbone, the electron polarization increases, which reduces the number of photons emitted from the chromophore group..$^{24}$ The exception is the circular plasmid DNA, in which the DOX molecules lost more than 37 -fold of the initial MB. The excitation of the DOX molecules depends on the proper orientation of the transition dipole moment (see Fig. 4(a)). The random orientation of the twisted linear DNA backbone makes the DOX MB change independent of fluorescence anisotropy. The DOX molecules that bind with circular DNA have a higher orientation order. As a result, they are more dependent on the dipole vector position of the DNA (see Fig. 4(b)). While in the second reaction, the similar MB loss shows that the rotation of the DOX molecules is not limited by the DNA chain. We infer that during the formation of DOX-(DOX-DNA) complexes, the interaction occurs between the aminoglycoside ring of the DOX molecules and the formed DOX-DNA complexes. Since the tetracenequinone chromophore group of the excess DOX can freely rotate, more photons can be emitted, leading to less and similar DOX MB in all DNA (see Fig. 4(c)).

\subsection{Equilibrium constants of DOX-DNA complex formation}

We have investigated that the DOX-DNA complex formation occurs in two reactions. Next, we determined the ratio of $\frac{\gamma_{1}}{\alpha}$ and $\frac{\gamma_{2}}{\alpha}$ as a ratio of the initial DOX MB loss in the first and second complex formations, respectively. Along with either eqn (3) or (5), we determined the value of $K_{1}$ and $K_{2}$. We kept a constant DOX concentration (40 nM) and varied the DNA concentration. In each titration point, we measured the count rate and plotted it as a function of initial DNA concentration $c_{\mathrm{bp}}$ (see Fig. 6(a)). Instead of fitting the function to the obtained data points with all released variables, we divided the series into previously specified regimes $\left(R_{1}<0.4<R_{2}\right)$. We determined $K_{1}$ by fitting eqn (3) only to the points corresponding to the regime $R_{1}<0.4$. After getting $K_{1}$, we obtained $K_{2}$ by fitting all data points with eqn (5). We performed the titration experiments on all DNA strands along with the corresponding MB and the results are shown in Fig. 6b. The average equilibrium constant is $(8.3 \pm 1.2) \times 10^{7} \mathrm{M}^{-1}$ in the first reaction and $(2.0 \pm$
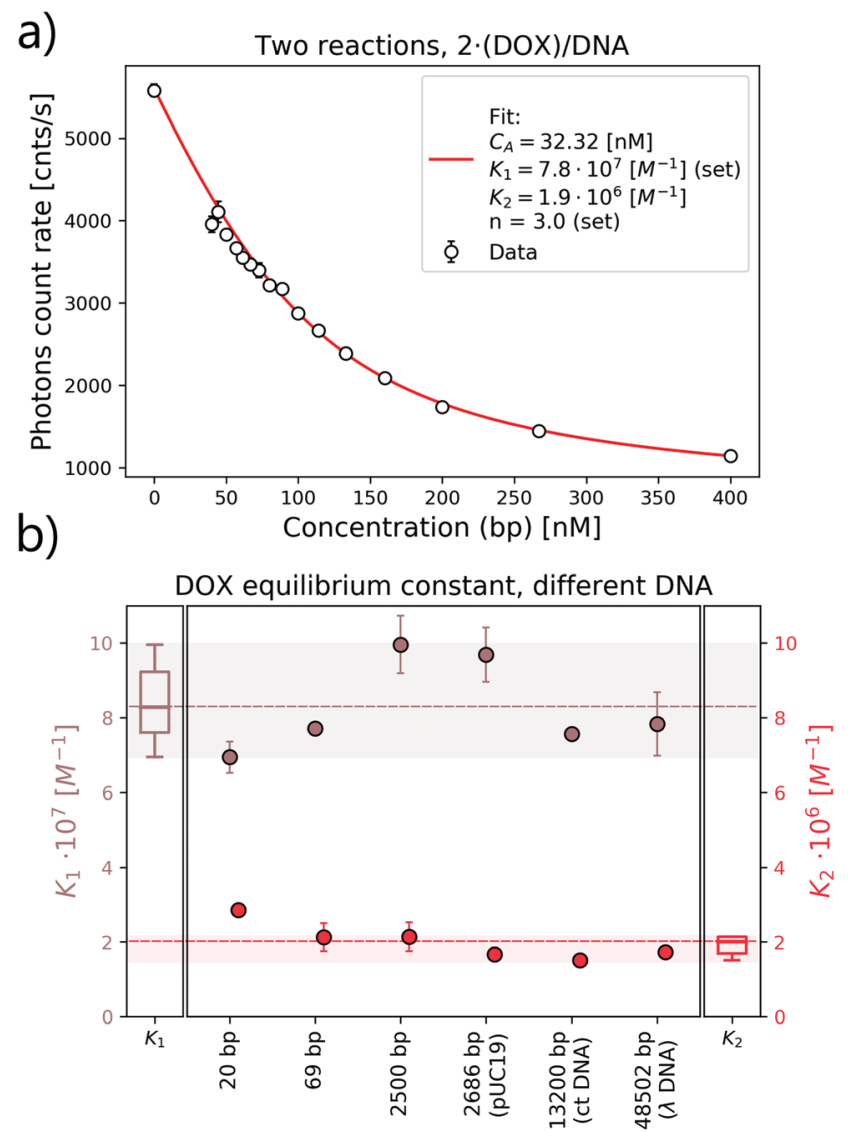

Fig. 6 Equilibrium constant determination of DOX-DNA interaction. (a) Equilibrium constant fit of DOX-DNA (69 bp) interaction including a two-reaction model. (b) List of the equilibrium constants of interactions between DOX and DNA with different lengths. 
$0.5) \times 10^{6} \mathrm{M}^{-1}$ in the second one. The difference in the equilibrium constant values between those reactions is around 40 times, whereas in Gracia's report, this difference is 200 times. $^{3}$ This discrepancy could be caused by the ionic strengths we used in our measuring conditions. In the previous reports of DOX-DNA interaction, when the sodium concentration increases from $2.5 \mathrm{mM}$ to $63 \mathrm{mM}$, the $K$ value decreases from $10^{8} \mathrm{M}^{-1}$ to $10^{7} \mathrm{M}^{-1} \cdot{ }^{2,3}$ The weaker the ionic strength, the larger the $K$ value. Since the ionic strength in our measurement was four times stronger than that in Garcia's report, ${ }^{3}$ it is reasonable for us to have a five times smaller $K$ than them.

The length or the structure of DNA does not affect the $K$ values, except for the $K_{2}$ obtained with 20 bp DNA (see statistical analysis in Fig. 6(b)). Our method is based on collecting the direct signal from the reaction pool, so that even a single photon change can be monitored. In comparison, the determination of $K$ by the FCS method is not based on observing the MB change in each reaction (even for long DNA). The FCS experiment is based on an analysis of the fluctuation of the fluorescence signal. Thus, the signal from the components with different $\mathrm{MB}$ would be averaged by the correlation function.

To ensure that the brightness analysis is observed only by the reaction between the DOX molecules and DNA, we tested the self-aggregation of DOX at our working concentration (40 $\mathrm{nM}$ ). We checked the possibility of DOX aggregation with UVvis spectroscopy. From the results of the absorption and emission spectra, we did not observe dimers or higher aggregates of DOX molecules at our working concentration (the details are shown in ESI $\dagger$ ).

\subsection{Determination of complex formation between DNA and DOX analogs}

Once the DOX-DNA interaction has been thoroughly investigated, we analysed daunorubicin (DNR), epirubicin (EPR), and idarubicin (IDR). Due to the similarity in structure, we expect that these three anthracyclines also attach to DNA with the same two reaction mechanisms as DOX. As there are limited references that state the bp number $(n)$ per binding site for those anthracyclines, we fit our curve with $n$ equal to $4.25 \pm$ $0.35,2.85 \pm 0.50$, and $3.50 \pm 0.71$ for DNR, EPR, and IDR, respectively. ${ }^{14,21,25,26}$ These values are in good agreement with the reported $n$ number $2-4$. To exclude the influence from structural differences, we monitored the MB change of each anthracycline in the interaction with 69 bp DNA and the results are shown in Fig. 7.

All three anthracyclines show a similar trend in MB change as DOX. Firstly, a strong MB loss (on average, 8.74 \pm 3.10 fold) in the first reaction and then a slight MB loss (averagely, $2.08 \pm$ 0.32 fold) in the second reaction. We determined the crossover points of the DNR-, EPR- and IDR-DNA interactions with values equal to $0.33 \pm 0.04,0.43 \pm 0.04$, and $0.30 \pm 0.00$, respectively. The results prove that the two reaction mechanisms are also present for DOX analogs.

Next, we determined $K_{1}$ and $K_{2}$ for those DOX analogs in the reaction with 69 bp DNA. Each measurement was repeated

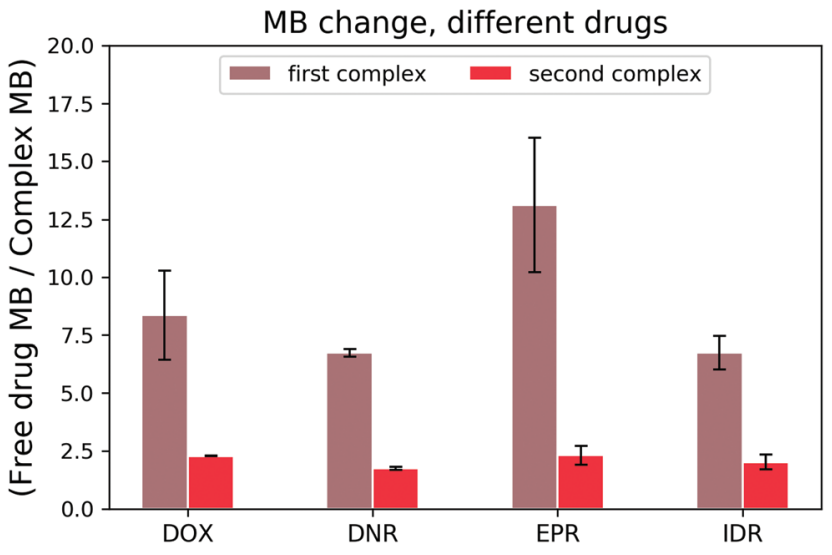

Fig. 7 Brightness loss of anthracyclines during each complex formation.

twice to exclude the random error. No self-aggregation of the drugs was observed under UV-vis spectroscopy measurements within the working range (the details can be seen in the $\mathrm{ESI} \dagger$ ). The obtained $K$ values for all four drugs are presented in Fig. 8.

The formation of the first complex of DOX, DNR, EPR, and IDR with DNA have $K_{1}$ equal to $(7.8 \pm 0.2) \times 10^{7} \mathrm{M}^{-1},(7.7 \pm 1.1)$ $\times 10^{7} \mathrm{M}^{-1},(7.5 \pm 0.9) \times 10^{7} \mathrm{M}^{-1}$, and $(4.8 \pm 0.2) \times 10^{7} \mathrm{M}^{-1}$, respectively. In the second reaction, DOX, DNR, EPR, and IDR have $K_{2}$ equal to $(2.1 \pm 0.4) \times 10^{6} \mathrm{M}^{-1},(9.8 \pm 1.0) \times 10^{5} \mathrm{M}^{-1}$, $(1.6 \pm 0.0) \times 10^{6} \mathrm{M}^{-1}$, and $(1.5 \pm 0.1) \times 10^{6} \mathrm{M}^{-1}$, respectively.

Determination of the reaction mechanism using the brightness analysis method was possible due to the significant difference in the strength of the interaction and brightness between the formed complexes of the individual reactions. The observation of the inflection point during the determination of the complex MB was a crucial part of the research. We expect no significant differences in the association/disassociation kinetics among those drugs in the binding with DNA based on the collected data during the analysis of the four most popular anthracyclines.

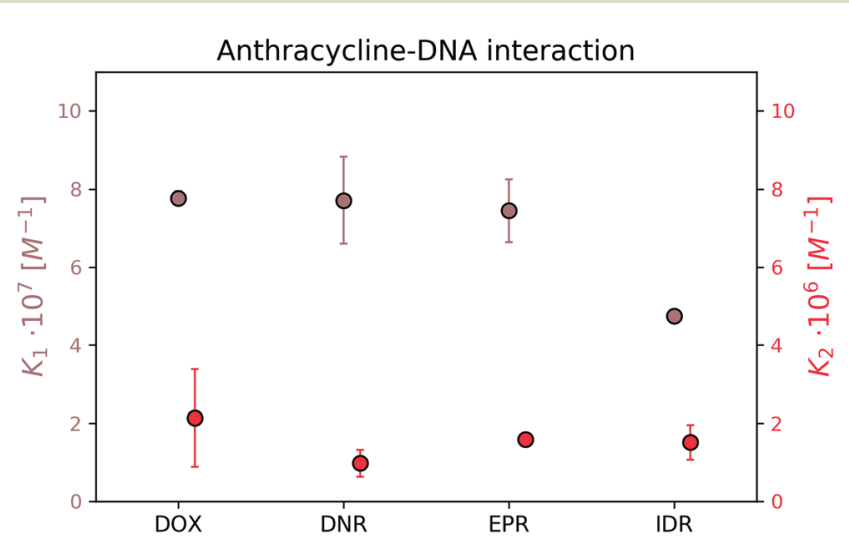

Fig. 8 Equilibrium constants for complexes of DOX analogs and DNA. 


\section{Conclusions}

We applied the molecular brightness method to determine the equilibrium constant of the anthracycline-DNA interaction at the nanomolar concentration scale. We tested four of the most used drugs in anticancer therapy: DOX, DNR, EPR, and IDR. Regardless of the low quantum yield of these anthracyclines, the method was sensitive enough to confirm the presence of two reaction mechanisms with DNA. The first reaction (i.e., drug-DNA) dominates when there are plenty of binding sites for the drug molecules to intercalate into. The second complex (i.e., drug to drug-DNA) is formed as soon as the first drug intercalates into DNA. The difference in $K$ of these two reactions is around forty times. We proposed the binding mechanism of the drugs in the second complex formation: the drug molecule anchors with aminoglycoside ring to the first formed complex. The provided data about the mechanism of the association/disassociation kinetics of DOX and its analogs can be used as a reference in the future use of those drugs. We hope that the simple principle of this method can be applied as an initial method in biochemical measurements. We also aim to develop potential methods for the selection of the most stable novel synthesized drug precursor complexes, the design of nano-drug delivery system, and the kinetic studies of in vivo biochemical reactions.

\section{Conflicts of interest}

There are no conflicts to declare.

\section{Acknowledgements}

This work was supported by the National Science Centre, Poland, within the grant Maestro UMO-2016/22/A/ST4/ 00017. Y. Z. acknowledges the support from the European Union's Horizon 2020 research and innovation program under the Marie Skłodowska-Curie grant agreement no. 711859 (CO-FUND Names project) and the financial resources from the Ministry of Science of Poland for science in the years 2017-2021 awarded for the implementation of an international co-financed project.

\section{References}

1 WHO, Mental and Holistic Health: Some International Perspectives, 2019, 119-134.

2 S. S. Tartakoff, J. M. Finan, E. J. Curtis, H. M. Anchukaitis, D. J. Couture and S. Glazier, Org. Biomol. Chem., 2019, 17, 1992-1998.
3 C. Pérez-Arnaiz, N. Busto, J. M. Leal and B. García, J. Phys. Chem. B, 2014, 118, 1288-1295.

4 M. Airoldi, G. Barone, G. Gennaro, A. M. Giuliani and M. Giustini, Biochemistry, 2014, 53, 2197-2207.

5 P. Changenet-Barret, T. Gustavsson, D. Markovitsi, I. Manet and S. Monti, Phys. Chem. Chem. Phys., 2013, 15, 29372944.

6 X. Zhang, A. Poniewierski, K. Sozański, Y. Zhou, A. Brzozowska-Elliott and R. Holyst, Phys. Chem. Chem. Phys., 2019, 21, 1572-1577.

7 P. Schwille and E. Haustein, Spectroscopy, 2001, 94(22), $1-33$.

8 D. A. Gewirtz, Biochem. Pharmacol., 1999, 57, 727-741.

9 B. Jawad, L. Poudel, R. Podgornik, N. F. Steinmetz and W. Y. Ching, Phys. Chem. Chem. Phys., 2019, 21, 3877-3893.

10 A. R. Rubio, N. Busto, J. M. Leal and B. García, RSC Adv., 2016, 6, 101142-101152.

11 J. V. McGowan, R. Chung, A. Maulik, I. Piotrowska, J. M. Walker and D. M. Yellon, Cardiovasc. Drugs Ther., 2017, 31, 63-75.

12 Z. Chen, G. Liu, M. Chen, X. Chen, M. Wu and X. Chen, Comb. Chem. High Throughput Screening, 2010, 13, 383-392.

13 B. Doughty, Y. Rao, S. W. Kazer, S. J. Kwok, N. J. Turro and K. B. Eisenthal, J. Phys. Chem. B, 2013, 117, 15285-15289.

14 P. F. Fouzia, A. Nasima, R. Qureshi, J. Nowsherwan, A. Sultan, B. Nosheen and H. Rafique, PLoS One, 2018, 13, 1-19.

15 S. Charak, D. K. Jangir, G. Tyagi and R. Mehrotra, J. Mol. Struct., 2011, 1000, 150-154.

16 R. Hajian, E. Ekhlasi and R. Daneshvar, E-J. Chem., 2012, 9, 1587-1598.

17 C. Ozluer and H. E. S. Kara, J. Photochem. Photobiol., B, 2014, 138, 36-42.

18 S. Charak and R. Mehrotra, Int. J. Biol. Macromol., 2013, 60, 213-218.

19 K. Bielec, G. Bubak, T. Kalwarczyk and R. Holyst, J. Phys. Chem. B, 2020, 124, 1941-1948.

20 H. Sierra, M. Cordova, C. S. J. Chen and M. Rajadhyaksha, J. Invest. Dermatol., 2015, 135, 612-615.

21 F. Barcelo, J. Martorell, F. Gavilanes and J. M. GonzalezRos, Biochem. Pharmacol., 1988, 37, 2133-2138.

22 Y. Wang and Z. Xu, RSC Adv., 2015, 6, 314-322.

23 D. Agudelo, P. Bourassa, G. Bérubé and H. A. Tajmir-Riahi, Int. J. Biol. Macromol., 2014, 66, 144-150.

24 B. Göhler, V. Hamelbeck, T. Z. Markus, M. Kettner, G. F. Hanne, Z. Vager, R. Naaman and H. Zacharias, Science, 2011, 331, 894-897.

25 S. R. Byrn and G. D. Dolch, J. Pharm. Sci., 1978, 67, 688693.

26 G. Goluński, A. Borowik, A. Lipińska, M. Romanik, N. Derewońko, A. Woziwodzka and J. Piosik, Bioorg. Chem., 2016, 65, 118-125. 